\title{
Rapid Identification of Pathogens from Positive Blood Cultures by Multiplex PCR using the FilmArray System
}

\author{
Anne J. Blaschke, MD, PhD ${ }^{1,}{ }^{*}$, Caroline Heyrend, BSc ${ }^{1,2}$, Carrie L. Byington, MD ${ }^{1}$, Mark A. \\ Fisher, $\mathrm{PhD}^{3,4}$, Elizabeth Barker, BSc ${ }^{2}$, Nicholas F. Garrone, PhD $^{2}$, Stephanie A. Thatcher, \\ MS $^{2}$, Andrew T. Pavia, MD ${ }^{1}$, Trenda Barney, MT $^{5}$, Garrison D. Alger, MLS ${ }^{5}$, Judy A. Daly, \\ PhD $^{3,5}$, Kirk M. Ririe, BSc ${ }^{2}$, Irene Ota, PhD $^{2}$, and Mark A. Poritz, PhD ${ }^{2}$ \\ ${ }^{1}$ University of Utah, Department of Pediatrics, Salt Lake City, Utah \\ ${ }^{2}$ Idaho Technology, Inc., Salt Lake City, Utah \\ ${ }^{3}$ Department of Pathology, University of Utah, Salt Lake City, Utah \\ ${ }^{4}$ Associated Regional and University Pathologists, Salt Lake City, Utah \\ ${ }^{5}$ Primary Children's Medical Center Microbiology Laboratory, Salt Lake City, UT
}

\section{Abstract}

Sepsis is a leading cause of death. Rapid and accurate identification of pathogens and antimicrobial resistance directly from blood culture could improve patient outcomes.

The FilmArray® (FA; Idaho Technology, Inc., Salt Lake City, UT) Blood Culture (BC) panel can identify $>25$ pathogens and 4 antibiotic resistance genes from positive blood cultures in 1 hour. We compared a development version of the panel to conventional culture and susceptibility testing on 102 archived blood cultures from adults and children with bacteremia. Of 109 pathogens identified by culture, $95 \%$ were identified by FA. Among 111 prospectively collected blood cultures, the FA identified 84 of 92 pathogens (91\%) covered by the panel. Among 25 Staphylococcus aureus and 21 Enterococcus species detected, FA identified all culture-proven MRSA and VRE.

The FA BC panel is an accurate method for the rapid identification of pathogens and resistance genes from blood culture.

\section{Keywords}

polymerase chain reaction; sepsis; bacteremia; blood culture; molecular diagnostics; bloodstream infection; FilmArray

\footnotetext{
() 2012 Elsevier Inc. All rights reserved.

*Correspondence to be sent to: Anne J. Blaschke, MD, PhD, Department of Pediatrics, Division of Pediatric Infectious Diseases, P.O Box 581289, Salt Lake City, UT 84158, (801) 581-6791 (phone), (801) 585-3789 (fax), anne.blaschke@ hsc.utah.edu.

Portions of this work were presented as a poster presentation at the American Society for Microbiology Annual Meeting, 2011 (New Orleans, LA)

Conflict of Interest: CH, EB, NFG, ST, GDA, KMR, IO and MAP are or were at the time of the study employees of Idaho Technology, Inc. AJB, CLB, JAD and ATP collaborate with Idaho Technology, Inc., on several NIH and CDC-funded projects (see funding above).

Publisher's Disclaimer: This is a PDF file of an unedited manuscript that has been accepted for publication. As a service to our customers we are providing this early version of the manuscript. The manuscript will undergo copyediting, typesetting, and review of the resulting proof before it is published in its final citable form. Please note that during the production process errors may be discovered which could affect the content, and all legal disclaimers that apply to the journal pertain.
} 


\section{Introduction}

Sepsis is a leading cause of death in U.S. adults and children (Kochanek, et al., 2011). Prompt initiation of appropriate antimicrobial therapy is associated with improved outcomes (Bochud, et al., 2001; Dellinger, et al., 2008; Kumar, 2009). Definitive identification of the causative organism through traditional culture methods often requires $24-72$ hours. This delay can lead to administration of either inadequate or overly broad antimicrobial therapy. Inappropriate antimicrobial selection may result in therapy-related complications, emergence of antimicrobial resistance, and increases in patient morbidity, mortality, and costs (Bauer, et al., 2010; Bochud, et al., 2001; Dellinger, et al., 2008; Kumar, 2009).

Continuously monitoring automated blood culture systems have enabled the detection of most blood stream pathogens within 24 hours of inoculation (Cockerill, et al., 2004). Automated systems for identification and susceptibility testing are available, however the organism must be isolated in pure culture prior to using them (Jorgensen and Ferraro, 2009). This requires a minimum of 12-24 hours after the blood culture becomes positive, and methods to improve bacterial growth rates are lacking (Mancini, et al., 2010).

Identification of pathogens directly from positive blood culture is one proposed solution. Ideally for such identification, rapid, species-specific identification of a wide range of pathogens would be provided. For PCR-based systems, this requires a high level of multiplexing that has been difficult to achieve (Mackay, 2004; Persson, et al., 2005). Here we describe the preliminary development of such an assay based on Idaho Technology's FilmArray ${ }^{\circledR}$ Platform (Poritz, et al., 2011), a closed diagnostic system allowing high-order multiplex PCR analysis with automated read-out of results. The assay described can identify 15 species-specific and 6 genus level targets, encompassing almost 95 percent of organisms commonly found in positive blood cultures (Biedenbach, et al., 2004). Four antibiotic resistance determinants are also included. Design of the assay panel as well as initial testing of a development version with clinical samples is presented.

\section{Materials and Methods}

The Institutional Review Boards of the University of Utah and Primary Children's Medical Center (PCMC, Salt Lake City, UT) approved this study. Informed consent was waived.

\section{FilmArray®}

The FDA approved FilmArray (FA) System has been described (Poritz, et al., 2011). Briefly, it is a closed diagnostic system that combines nucleic acid extraction from clinical specimens, high-order nested multiplex PCR, and post-PCR DNA melt curve analysis.

\section{Panel Design}

Nested and nested multiplex PCR-The FA system performs a "nested multiplex" PCR (nmPCR) within the instrument (Poritz, et al., 2011). Briefly, nmPCR is a 2-stage PCR in which the first-stage reaction is a multiplex that produces a 200-500 bp amplicon for each target. The second-stage primers are "nested" within this amplicon and amplify a shorter product. Nested PCR provides increased sensitivity compared with conventional PCR; the specificity is similar to probe-based assays (Narayanan, 1996). Assays were developed to function within the context of nmPCR, with first-stage multiplex amplification performed for 20 cycles and the array of second stage singleplex nested PCRs performed for 25 cycles.

Panel Design-Organisms were chosen for the BC panel based on laboratory data provided by our collaborating microbiology laboratory director (JAD) and through review of 
SENTRY data regarding organism prevalence in blood cultures (Biedenbach, et al., 2004). Both organisms with a high frequency and low pathogenicity [i.e. coagulase-negative staphylococci (CONS)] and those with a low frequency and high pathogenicity (i.e., Neisseria meningitidis) were included. The panel used in the studies presented here includes 15 species-specific targets and 6 genus-level assays. This panel (Table 1) represents $\sim 90$ $95 \%$ of all pathogens identified from participating laboratories and the SENTRY database.

Antibiotic resistance targets were chosen based on frequency of detection, implications for change in antibiotic therapy, and the likelihood of a direct correlation between gene presence and antibiotic resistance. The genes mecA, van $A$, van $B$ and the Klebsiella pneumoniae carbapenemase ( $b l a_{\mathrm{KPC}}$ ) were included in the panel (Cole, et al., 2009; Fluit, et al., 2001). In the version of the panel used for clinical validation, the vanC gene was also included, however this gene was removed from the panel used for the prospective study due to its low prevalence and association with a variable resistance phenotype (Clark, et al., 1998).

For species-specific bacterial targets such as virulence factors, genes were chosen based on a review of the literature. For genus (i.e. "Streptococcus spp.") or family (i.e.

"Enterobacteriaceae") level assays, conserved housekeeping genes were targeted and degenerate primers were designed to encompass important species within the group. For example, the Streptococcus species assay targets the RNA polymerase $\beta$ subunit (rpoB) gene and was designed to capture 7 species in the target alignment. Primers for antibiotic resistance genes are all located in conserved regions so that only one set contains degenerate bases ( van $A$ gene; 2-fold degeneracy). The developmental panel used in these studies includes 50 first-stage primers in the multiplex and 65 second-stage singleplex PCRs.

Primer Design-Gene sequences were obtained through the National Center for Biotechnology Information's GenBank Database (Benson, et al., 2011; Sayers, et al., 2011). Alignments were created and analyzed using Lasergene ${ }^{\circledR}$ software (DNASTAR, Madison, WI). Primers were designed using LightCycler ${ }^{\circledR}$ Primer Probe Design Software (Roche Applied Science, Indianapolis, IN). Degenerate primers were used in primer design in cases where species were divergent within the gene target and in genus-level assays.

\section{Assay optimization}

Assay optimization was performed as previously described for FilmArray system development (Poritz, et al., 2011).

Bacteria and Yeast Isolates-Bacteria and yeast isolates were obtained from the PCMC microbiology laboratory and from the University of Utah's Associated Regional and University Pathologists (ARUP) Laboratories (Salt Lake City, UT). Clinical isolates were identified by standard microbiologic methods (see below). In addition isolates were obtained from the American Type Culture Collection (ATCC; Manassas, VA) and from JMI Laboratories (North Liberty, IA).

Assay validation-All assays were validated for sensitivity and specificity as individual real-time PCR reactions and in the FA pouch with bacterial isolates and their extracted nucleic acids. For the Blood Culture (BC) panel described, targets analyzed in the FA included known positives for each pathogen at levels of approximately $100-10,000$ genomes per pouch based on DNA fluorimeter measurements (Qubit Fluorimeter, Life Technologies, Grand Island, NY). Testing was also performed with bacteria/yeast isolates (2-3 colonies resuspended in $500 \mu \mathrm{l}$ of the sample buffer). For species-specific assays, testing was performed with at least 5 different isolates of each organism. Specific 
amplification of targets was evaluated by testing against "nearest neighbors" (ie, testing of the $S$. pneumoniae assay against viridans streptococci, $S$. pyogenes and $S$. agalactiae). Genus or group level assays were tested with 1-5 isolates of each target organism within the group. All assays in the developmental panel had a sensitivity of $<100$ genomes per pouch and $100 \%$ species-or genus-level specificity for bacterial isolates tested (data not shown).

\section{Blood Culture Analyses}

Conventional microbiology at Primary Children's Medical Center (PCMC) and the University of Utah's Associated Regional and University Pathologists (ARUP) Laboratories-Both PCMC and ARUP are Clinical Laboratory Improvement Amendments (CLIA) certified. In both laboratories, blood culture is performed using the BACTEC automated blood culture system (BD Diagnostic Systems, Franklin Lakes, NJ). Cultures signaling positive are removed immediately from the instrument and Gram-stain is performed within 15 minutes. Isolates of bacteria and yeast are grown on solid media and at PCMC are identified by VITEK® (bioMerieux, Inc., Durham, NC) and by bench top biochemical testing. At ARUP, isolates are identified by Phoenix automated identification system (BD Diagnostic Systems), bench top biochemical testing and/or 16S rRNA gene sequencing. Antibiotic susceptibility is performed at PCMC by VITEK and by E-Test ${ }^{\circledR}$ (bioMerieux, Inc.) and at ARUP by the Phoenix (BD Diagnostic Systems), manual broth microdilution (Trek Diagnostic Systems, Inc., Cleveland, OH), and/or E-Test@. PCR is performed at ARUP for identification of the $m e c A$ gene.

Collection of Blood Culture Samples- $1.5 \mathrm{ml}$ samples from blood culture bottles (BD Diagnostic Systems, Franklin Lakes, NJ) signaling positive were collected from PCMC and from ARUP Laboratories. Sample collection was initiated at the onset of panel development and samples were stored frozen at $-80^{\circ} \mathrm{C}$ until testing.

\section{Validation Study}

A validation designed to demonstrate the accuracy of pathogen identification from clinical blood cultures was performed using blood culture specimens obtained and frozen in 20062007 from PCMC. De-identified samples were chosen to represent the majority of pathogens on the development version of the FA BC panel by an investigator (AJB) aware of the culture result, and were tested using the FA by an investigator who was blinded to the culture results $(\mathrm{CH})$.

\section{Prospectively Collected Sequential Samples}

The utility of the panel for identification of pathogens commonly encountered in the clinical setting was evaluated through prospectively collected sequential samples from all positive blood cultures obtained between June and August 2010 from PCMC and ARUP. As the intended use of the panel in the clinical laboratory is to test samples that signal positive in the automated blood culture system, negative blood cultures were not specifically tested. Testing was performed, however, if a culture bottle signaled positive, even if organisms were not seen on Gram stain. When more than one sample was collected from a single patient, only the first positive sample was analyzed. Samples were de-identified and frozen within 2 hours of the blood culture signaling positive. All investigators testing samples on the FA were blinded to culture results.

\section{Data Analysis}

FA Results-Automated calls for the assays in the FA BC pouch were made using a beta version of the FA software (Poritz, et al., 2011) tuned for the BC pouch. Two investigators 
(AJB and $\mathrm{CH}$ ) confirmed all automated calls based on examination of amplification and melt curves.

In this developmental pouch version we observed low-level background contamination of reagents and the pouch. This is a common problem of broad-range PCR-based bacterial detection systems (Chang, et al., 2011; Corless, et al., 2000; Millar, et al., 2002; Philipp, et al., 2010; Sontakke, et al., 2009). Contaminants demonstrated late amplification [after 22 cycles in the second stage PCR (36 cumulative first + second stage cycles)] but generated positive calls by the software. Contaminants were consistent across a production lot of pouches and were identified by positive calls in the 3-5 negative control (water) runs performed for each lot. With this information, positive software calls were classified as contamination if a run was positive for a known lot-contaminating organism and the cycle crossing point was $>22$ cycles in the second-stage PCR.

Statistical analysis-For both the validation and prospective studies sensitivity and specificity were calculated for the BC panel overall and for each pathogen on the BC panel independently. $95 \%$ confidence intervals were calculated according to the efficient-score method (corrected for continuity) (Newcombe, 1998). Blood culture was considered the gold standard. A FA test positive was identified when the FA BC assay was positive for a pathogen, and a true positive was identified when the blood culture was positive for the same pathogen. For specificity calculation, all samples negative for a particular pathogen were included.

\section{Results}

\section{Clinical validation study using retrospectively collected positive blood cultures}

Initial validation of the $\mathrm{BC}$ panel was performed on 102 clinical blood culture samples selected to be positive for organisms representing the majority of panel pathogens. These samples were tested retrospectively. Sixteen different pathogens were represented. No clinical specimens containing Listeria monocytogenes, Acinetobacter baumanii or Candida spp. were available in this study set and thus these are not included in Table 2. Due to the wide confidence intervals around the point estimates of sensitivity, these results are reported with an asterisk for organisms with $<5$ clinical samples available (Table 2). Organisms containing $\operatorname{van} A / B / C$ and $m e c A$ were tested, but no organisms were found either by culture or FA to be carbapenem-resistant or to contain bla ${ }_{\mathrm{KPC}}$.

From 102 clinical specimens, 109 pathogens were identified by culture ( 7 cultures were positive for more than one organism). Of the 109 pathogens identified by culture, 104 (95\%) were also identified by FA. Three pathogens identified by FA were not identified by culture. Sensitivity for each pathogen ranged from $83-100 \%$ and specificity was $99 \%$ or greater for all. (Table 2)

The culture-positive/FA-negative discordant samples were analyzed using standard PCR. In 2 cases the specimen was also negative using both an alternative organism-specific assay as well as broad-range (16S) PCR, suggesting a mislabeled or incorrect culture sample. In two discordant samples in which culture and FA identified different pathogens, standard PCR and sequencing demonstrated that the organism identified by FA was correct. In one case of a culture-identified CONS missed by FA, the organism could not be detected using alternative PCR strategies. In the case of a FA-identified CONS not identified by culture, sequencing confirmed the presence of $S$. epidermidis.

Antibiotic resistance testing demonstrated good concordance with conventional susceptibility data. Thirteen $S$. aureus were identified by both culture and FA, of which 4 
were methicillin-resistant $S$. aureus (MRSA) by susceptibility testing. The FA appropriately identified all $S$. aureus as MSSA (mecA negative) or MRSA ( $m e c A$ positive). Of 13 enterococci identified, one was found by culture to be vancomycin-resistant (VRE) and positive for $v a n A / B / C$ by FA. Two enterococci tested susceptible to vancomycin in the microbiology laboratory but tested positive for $v a n A / B / C$ by FA. One of these isolates was identified by culture, standard PCR, and sequencing as E. faecium. PCR and sequencing also demonstrated the van $A$ gene to be present, with $E$. faecium-specific van $A$ polymorphisms based on alignment to sequences in GenBank. The other sample, identified by culture as vancomycin-susceptible $E$. faecalis, was identified by sequencing as $E$. casseliflavus containing the $\operatorname{vanC}$ gene.

\section{Prospective analysis of sequentially collected samples}

One hundred eleven blood culture samples were tested in this study (Table 3). Ninety-three were culture-positive. Eighteen were Gram-stain and culture-negative despite signaling positive on the automated blood culture machine. The 93 positive cultures grew 99 organisms (6 dual-positive cultures), of which 92 (93\%) were pathogens included in the BC panel. Seven were positive for non-panel organisms [Micrococcus spp. $(\mathrm{n}=3)$;

Corynebacterium spp., Fusobacterium nucleatum, Propionibacterium acnes, Diptheroids (n=1 each)]. In this study set there were no samples positive by culture or FA for $L$. monocytogenes, E. cloacae, $N$. meningitidis or $H$. influenzae and no pathogens contained bla $\mathrm{KPC}$.

The FA identified 84 of the 92 (91\%) BC pathogens identified by culture. Sensitivity varied by organism, and some organisms were rarely seen, but all assays had high specificity (Table 3). The overall likelihood ratio positive for the test was 4 and the likelihood ratio negative was 0.11 .

In two instances, a culture was positive for two different organisms of the same genus [2 different species of viridans streptococci $(n=1)$ and 2 species of CONS $(n=1)]$. These could not be resolved by the genus-level PCR and were not counted as discordant results. FA accurately identified all $S$. aureus as MSSA or MRSA and all enterococci as susceptible or resistant to vancomycin. Except for one coagulase-negative staphylococcus detection by FA, all culture-negative samples and those positive for pathogens not in the $\mathrm{BC}$ panel were negative by FA.

FA did not identify eight pathogens identified in the 92 blood cultures. In six instances $(6.5 \%)$ these were shown to be FA false-negatives. In three of these cases (2 yeast and 1 Enterococcus spp.) standard PCR with an increased number of cycles identified the organism. In two cases, samples positive for CONS by culture were negative by the CONS FA assay, although the mec $A$ gene PCR was positive. In one case, $S$. marcescens identified in culture was not detected by FA or with an increased number of cycles in standard PCR, but was found by both culture and PCR in subsequent blood cultures from the same patient. Three of the 8 (37.5\%) missed organisms were in dual-positive cultures; FA correctly identified one of the pathogens but did not detect the second. In 2 instances, primer crossreactivity resulted in misidentification of organisms: in one culture positive for Salmonella enterica the FA misidentified the organism as Klebsiella oxytoca; in another case, FA identified CONS as well as $S$. aureus in a culture positive for $S$. aureus alone. The CONS identification was confirmed to be a cross-amplification of this particular $S$. aureus by the CONS primers.

Seven pathogens identified by FA were not identified by culture. While 2 were the result of the primer cross-reactivity described above, the other five organisms identified by FA but not by culture were likely true positives. In one case, Gram stain of BACTEC bottles 
showed Gram-negative rods, but no growth was detected on culture. FA identified two organisms, E. coli and Acinetobacter baumanii, both of which were confirmed by standard PCR and sequencing. In two cases, the FA identified a second organism in cultures that yielded growth for only one organism. In the first, $S$. aureus was detected by FA in a sample also culture and PCR positive for GAS. In the second, S. mitis/oralis was detected by FA in a sample also culture and PCR positive for CONS. In each case, the presence of both pathogens was confirmed by PCR and sequencing. Finally the FA identified a CONS that was confirmed by PCR and sequencing to be present in a clinical specimen that was negative by culture.

\section{Discussion}

Rapid identification of organisms causing sepsis is critical to reducing inappropriate antibiotic use and infection-related morbidity and mortality. Here we describe preliminary development and clinical testing of a novel molecular diagnostic test for the identification of organisms in positive blood culture based on the FilmArray® System (Poritz, et al., 2011). The FA BC panel identifies a wide range of bacterial and fungal pathogens, accounting for 93\% of organisms isolated from clinical specimens in our study. In addition, the panel identifies select antibiotic resistance genes, including those responsible for resistance to methicillin, vancomycin and carbapenems. Identification of pathogens and resistance markers was achieved from clinical samples with good sensitivity and specificity for all targets. An analysis of prospectively obtained blood cultures demonstrated accurate identification of $91 \%$ of pathogens and 100\% of MRSA and VRE. When fully developed, this technology has the potential to contribute significantly to improvements in the management of adults and children with sepsis.

Rapid molecular identification of organisms from positive blood cultures is increasingly available in clinical laboratories. Both PCR and probe-based platforms are commercially available, but all have limitations. In the U.S., the Xpert ${ }^{\circledR}$ MRSA/SA BC assay (Cepheid, Sunnyvale, CA) and the fluorescence in situ hybridization method employing novel peptidenucleic acids as probes (PNA-FISH®; AvanDx, Woburn, MA) are available, but both target limited numbers of organisms or require several panels (Hensley, et al., 2009; Morgan, et al., 2010; Shepard, et al., 2008). In Europe, bacterial detection methods from blood culture based on broad-range PCR are available that target a larger range of organisms in a single assay, but downstream identification using ELISA (Hyplex®; Cepheid Benelux, Bouwel, Belgium) or microarray hybridization (Prove-it ${ }^{\mathrm{TM}}$ Sepsis; Mobidiag, Helsinki, Finland) is required. One of the most comprehensive systems under investigation is based on matrixassisted laser desorption ionization time-of-flight (MALDI-TOF) mass spectrometry (La Scola and Raoult, 2009; Stevenson, et al., 2010). MALDI-TOF has the advantage of rapid turn-around time, modest sample preparation, and the ability to directly identify a large range of microorganisms. Disadvantages include the high start-up costs associated with the purchase of mass-spectrometry equipment and training of personnel.

Identification of blood culture pathogens using the FilmArray® System's BC panel has several advantages. A particular strength is the large number of targets, all evaluated in a single test, covering the majority of organisms detected in both adult and pediatric blood cultures. In this study, organisms identified by blood culture but not contained in the FA BC panel primarily included those often considered contaminants, such as diphtheroids and micrococcus. In addition, the FA System performs all steps of the assay, from nucleic acid extraction to interpretation of amplification data in closed system using a single pouch on a minimally processed clinical sample. The laboratory procedures required are not technologically complex and can be performed by persons who do not have training in molecular techniques (Poritz, et al., 2011). 
Our clinical validation study of 102 retrospectively-collected blood culture samples demonstrated accurate identification of most pathogens. In the primary analysis there were 8 discordant identifications ( 5 culture+/FA-; 3 culture-/FA+), however all but one identification (of CONS) could be resolved in favor of the FA. Specificity was high for all pathogens, including species-specific and genus-level targets. Specificity was comparable to that of the VITEK2 for cultured organisms, for which the accuracy of identification ranges from 92-97\% (Abele-Horn, et al., 2006; Nonhoff, et al., 2005; O'Hara and Miller, 2003; Rennie, et al., 2008).

The FA BC panel also contains antibiotic resistance markers. MRSA is a well-described health-threat (Klevens, et al., 2007), and a number of commercially available panels can distinguish MRSA from methicillin-susceptible strains through the identification of the $m e c A$ gene that is also identified in our panel. While in Europe a Nanosphere panel (Verigene System, Nanosphere, Northbrook IL) is available for detection of the primary genetic determinants of vancomycin-resistance in enterococci [ $\operatorname{van} A$ and $\operatorname{van} B$ ] (Courvalin, 2006), no commercially available panels approved for blood culture in the U.S. contain assays for $\operatorname{van} A$ and $\operatorname{van} B$ or carbapenem-resistance in enteric organisms [bla $a_{\mathrm{KPC}}$ ] (Nordmann, et al., 2009).

In choosing resistance targets for the $\mathrm{BC}$ panel, we chose genes in which detection of the resistance marker almost always indicates a phenotypically drug-resistant organism. We had one true genotype-phenotype discordance detected with regard to vancomycin susceptibility in our validation study: an $E$. faecium that was PCR positive for $\operatorname{van} A$, yet phenotypically vancomycin-susceptible. This discordance has been rarely reported (Choi, et al., 2011).

Our prospective study of consecutively collected samples was designed to evaluate the performance of the system on an unbiased selection of positive blood cultures. Overall, the system performed well, although the sensitivity was lower for Serratia marcescens (1/2), broad Enterobacteriaceae detection (3/4) and yeasts (4/6). The small numbers of samples preclude accurate assessment of the sensitivity for these organisms, however for others, sensitivity was good (88-100\%). Specificity was $>98 \%$ for all organisms.

There are limitations to the $\mathrm{BC}$ system at this point in development. While comprehensive, the FA BC panel does not detect all possible organisms. This reflects both a limitation in the number of assays that could be placed into the multiplex, and a conscious decision to primarily target organisms that occur at a high enough frequency to support FDA clearance. Sensitivity was not an issue for most organisms, however two of six Candida species detected in culture were not detected by FA. We hypothesized that yeast might signal "positive" at lower titers in automated blood culture systems. We performed growth studies in which the concentration of yeast at the time of culture positivity ranged from $10^{3}-10^{7}$ $\mathrm{CFU} / \mathrm{ml}$ rather than the $10^{7}-10^{11}$ seen with bacteria (Tan, et al., 2008) similar to another published report (Chang, et al., 2000). For this reason we are evaluating multi-copy rather than single-copy targets for yeast detection.

Polymicrobial culture was the most common reason that a cultured organism went undetected by PCR. Approximately $7 \%$ of blood cultures in the prospective study were polymicrobial, however in three of eight (38\%) instances in which the FA did not detect a pathogen, the blood culture was positive for two organisms. We speculate that in cultures positive for two organisms, one may grow more rapidly and trigger a positive signal in the culture system. At the time of the positive call, the second organism may be below the level of molecular detection.

As often reported for PCR-based detection systems that evaluate a broad range of bacteria, contamination of reagents and materials was an issue in this developmental version of the 
system (Chang, et al., 2011; Corless, et al., 2000; Millar, et al., 2002; Philipp, et al., 2010; Sontakke, et al., 2009). This is most often reported for 16S PCR-based detection, however in our system we have specific assays for potentially contaminating bacteria such as CONS and E. coli. At the level of research and development this was not a significant issue for evaluation of the sensitivity and specificity of the panel. In positive blood cultures, second stage crossing thresholds for culture-identified organisms were generally in the range of 1316 cycles. The level of contamination in the pouches was quite low, with second stage PCR crossing thresholds beyond 22 cycles [ 36 cumulative cycles] or signal only detected by the presence of a melting curve. Current development efforts are focused on eliminating detectible contamination through improvements in manufacturing as well as software cutoffs for commonly contaminating organisms. Overall system sensitivity must be maintained for the detection of yeasts and polymicrobial cultures as previously discussed.

A primary limitation of this study was the low number of samples positive for some organisms. While nucleic acid testing demonstrated good sensitivity and specificity of assays such as those for L. monocytogenes, $N$. meningitidis and H. influenzae, the low number of positive blood culture specimens makes clinical sensitivity hard to determine. In addition, there were no clinical specimens demonstrating carbapenem resistance or that were positive by PCR for bla $a_{\mathrm{KPC}}$. Additional studies, including at sites with higher rates of resistance will be necessary for clinical evaluation of these assays.

Overall the FA BC panel demonstrated accurate identification of pathogens found in positive blood cultures from both adult and pediatric patients. This panel identifies a larger range of pathogens than most currently available platforms in a system that is technically simple for the laboratory. The data presented here suggest that the FA BC system could be an important and useful tool for the management of bloodstream infection in the future.

\section{Acknowledgments}

This work was supported by: NIH/NIAID: 1K23AI079401 (AJB); 1U01AI082184 (AJB, ATP, JAD, IO); NIH/ NICHD K24HD047249, 1U01AI082482 (CLB), 1U01 AI074419 (CLB, MP, JAD); U01A1061611 (MAP, CLB); CDC: 1U18IP000303 (AJB, ATP, CLB) and NIH/NCRR UL1RR025764 (AJB, CLB).

\section{References}

Abele-Horn M, Stoy K, Frosch M, Reinert RR. Comparative evaluation of a new Vitek 2 system for identification and antimicrobial susceptibility testing of Streptococcus pneumoniae. Eur J Clin Microbiol Infect Dis. 2006; 25:55-7. [PubMed: 16424974]

Bauer KA, West JE, Balada-Llasat JM, Pancholi P, Stevenson KB, Goff DA. An antimicrobial stewardship program's impact with rapid polymerase chain reaction methicillin-resistant Staphylococcus aureus/S. aureus blood culture test in patients with S. aureus bacteremia. Clin Infect Dis. 2010; 51:1074-80. [PubMed: 20879856]

Benson DA, Karsch-Mizrachi I, Lipman DJ, Ostell J, Sayers EW. GenBank. Nucleic Acids Res. 2011; 39:D32-7. [PubMed: 21071399]

Biedenbach DJ, Moet GJ, Jones RN. Occurrence and antimicrobial resistance pattern comparisons among bloodstream infection isolates from the SENTRY Antimicrobial Surveillance Program (1997-2002). Diagn Microbiol Infect Dis. 2004; 50:59-69. [PubMed: 15380279]

Bochud PY, Glauser MP, Calandra T. Antibiotics in sepsis. Intensive Care Med. 2001; 27 (Suppl 1):S33-48. [PubMed: 11307369]

Chang HC, Chang JJ, Huang AH, Chang TC. Evaluation of a capacitance method for direct antifungal susceptibility testing of yeasts in positive blood cultures. J Clin Microbiol. 2000; 38:971-6. [PubMed: 10698982] 
Chang SS, Hsu HL, Cheng JC, Tseng CP. An efficient strategy for broad-range detection of low abundance bacteria without DNA decontamination of PCR reagents. PLoS One. 2011; 6:e20303. [PubMed: 21637859]

Choi HJ, Nam D, Peck KR, Song JH, Shin D, Ko KS. Loss of vancomycin resistance not completely dependent on the Tn1546 element in Enterococcus faecium isolates. Diagn Microbiol Infect Dis. 2011; 69:105-10. [PubMed: 21146722]

Clark NC, Teixeira LM, Facklam RR, Tenover FC. Detection and differentiation of vanC-1, vanC-2, and vanC-3 glycopeptide resistance genes in enterococci. J Clin Microbiol. 1998; 36:2294-7. [PubMed: 9666008]

Cockerill FR 3rd, Wilson JW, Vetter EA, Goodman KM, Torgerson CA, Harmsen WS, et al. Optimal testing parameters for blood cultures. Clin Infect Dis. 2004; 38:1724-30. [PubMed: 15227618]

Cole JM, Schuetz AN, Hill CE, Nolte FS. Development and evaluation of a real-time PCR assay for detection of Klebsiella pneumoniae carbapenemase genes. J Clin Microbiol. 2009; 47:322-6. [PubMed: 19036932]

Corless CE, Guiver M, Borrow R, Edwards-Jones V, Kaczmarski EB, Fox AJ. Contamination and sensitivity issues with a real-time universal 16S rRNA PCR. J Clin Microbiol. 2000; 38:1747-52. [PubMed: 10790092]

Courvalin P. Vancomycin resistance in gram-positive cocci. Clin Infect Dis. 2006; 42 (Suppl 1):S2534. [PubMed: 16323116]

Dellinger RP, Levy MM, Carlet JM, Bion J, Parker MM, Jaeschke R, et al. Surviving Sepsis Campaign: international guidelines for management of severe sepsis and septic shock: 2008. Crit Care Med. 2008; 36:296-327. [PubMed: 18158437]

Fluit AC, Visser MR, Schmitz FJ. Molecular detection of antimicrobial resistance. Clin Microbiol Rev. 2001; 14:836-71. [PubMed: 11585788]

Hensley DM, Tapia R, Encina Y. An evaluation of the advandx Staphylococcus aureus/CNS PNA FISH assay. Clin Lab Sci. 2009; 22:30-3. [PubMed: 19354026]

Jorgensen JH, Ferraro MJ. Antimicrobial susceptibility testing: a review of general principles and contemporary practices. Clin Infect Dis. 2009; 49:1749-55. [PubMed: 19857164]

Klevens RM, Morrison MA, Nadle J, Petit S, Gershman K, Ray S, et al. Invasive methicillin-resistant Staphylococcus aureus infections in the United States. JAMA. 2007; 298:1763-71. [PubMed: 17940231]

Kochanek KD, Xu J, Murphy SL, Minino AM, Kung H-C. Deaths: Preliminary Data for 2009. National Vital Statistics Reports. 2011; 59

Kumar A. Optimizing antimicrobial therapy in sepsis and septic shock. Crit Care Clin. 2009; 25:73351. viii. [PubMed: 19892250]

La Scola B, Raoult D. Direct identification of bacteria in positive blood culture bottles by matrixassisted laser desorption ionisation time-of-flight mass spectrometry. PLoS One. 2009; 4:e8041. [PubMed: 19946369]

Mackay IM. Real-time PCR in the microbiology laboratory. Clin Microbiol Infect. 2004; 10:190-212. [PubMed: 15008940]

Mancini N, Carletti S, Ghidoli N, Cichero P, Burioni R, Clementi M. The era of molecular and other non-culture-based methods in diagnosis of sepsis. Clin Microbiol Rev. 2010; 23:235-51. [PubMed: 20065332]

Millar BC, Xu J, Moore JE. Risk assessment models and contamination management: implications for broad-range ribosomal DNA PCR as a diagnostic tool in medical bacteriology. J Clin Microbiol. 2002; 40:1575-80. [PubMed: 11980924]

Morgan M, Marlowe E, Della-Latta P, Salimnia H, Novak-Weekley S, Wu F, et al. Multicenter evaluation of a new shortened peptide nucleic acid fluorescence in situ hybridization procedure for species identification of select Gram-negative bacilli from blood cultures. J Clin Microbiol. 2010; 48:2268-70. [PubMed: 20357212]

Narayanan S. Concepts, principles, and applications of selected molecular biology techniques in clinical biochemistry. Advances in Clinical Chemistry. 1996; 32:1-38. [PubMed: 8899069]

Newcombe RG. Two-sided confidence intervals for the single proportion: comparison of seven methods. Stat Med. 1998; 17:857-72. [PubMed: 9595616] 
Nonhoff C, Rottiers S, Struelens MJ. Evaluation of the Vitek 2 system for identification and antimicrobial susceptibility testing of Staphylococcus spp. Clin Microbiol Infect. 2005; 11:150-3. [PubMed: 15679491]

Nordmann P, Cuzon G, Naas T. The real threat of Klebsiella pneumoniae carbapenemase-producing bacteria. Lancet Infect Dis. 2009; 9:228-36. [PubMed: 19324295]

O'Hara CM, Miller JM. Evaluation of the Vitek 2 ID-GNB assay for identification of members of the family Enterobacteriaceae and other nonenteric gram-negative bacilli and comparison with the Vitek GNI+ card. J Clin Microbiol. 2003; 41:2096-101. [PubMed: 12734254]

Persson K, Hamby K, Ugozzoli LA. Four-color multiplex reverse transcription polymerase chain reaction--overcoming its limitations. Anal Biochem. 2005; 344:33-42. [PubMed: 16039598]

Philipp S, Huemer HP, Irschick EU, Gassner C. Obstacles of Multiplex Real-Time PCR for Bacterial 16S rDNA: Primer Specifity and DNA Decontamination of Taq Polymerase. Transfus Med Hemother. 2010; 37:21-8. [PubMed: 20737013]

Poritz MA, Blaschke AJ, Byington CL, Meyers L, Nilsson K, Jones DE, et al. FilmArray, an automated nested multiplex PCR system for multi-pathogen detection: development and application to respiratory tract infection. PLoS One. 2011; 6:e26047. [PubMed: 22039434]

Rennie RP, Brosnikoff C, Shokoples S, Reller LB, Mirrett S, Janda W, et al. Multicenter evaluation of the new Vitek 2 Neisseria-Haemophilus identification card. J Clin Microbiol. 2008; 46:2681-5. [PubMed: 18579712]

Sayers EW, Barrett T, Benson DA, Bolton E, Bryant SH, Canese K, et al. Database resources of the National Center for Biotechnology Information. Nucleic Acids Res. 2011; 39:D38-51. [PubMed: 21097890]

Shepard JR, Addison RM, Alexander BD, Della-Latta P, Gherna M, Haase G, et al. Multicenter evaluation of the Candida albicans/Candida glabrata peptide nucleic acid fluorescent in situ hybridization method for simultaneous dual-color identification of C. albicans and C. glabrata directly from blood culture bottles. J Clin Microbiol. 2008; 46:50-5. [PubMed: 17977998]

Sontakke S, Cadenas MB, Maggi RG, Diniz PP, Breitschwerdt EB. Use of broad range16S rDNA PCR in clinical microbiology. J Microbiol Methods. 2009; 76:217-25. [PubMed: 19046999]

Stevenson LG, Drake SK, Murray PR. Rapid identification of bacteria in positive blood culture broths by matrix-assisted laser desorption ionization-time of flight mass spectrometry. J Clin Microbiol. 2010; 48:444-7. [PubMed: 19955282]

Tan TY, Ng LS, Kwang LL. Evaluation of disc susceptibility tests performed directly from positive blood cultures. J Clin Pathol. 2008; 61:343-6. [PubMed: 17630402]

Diagn Microbiol Infect Dis. Author manuscript; available in PMC 2013 December 01. 
Table 1

Organisms Contained within the Developmental FilmArray BC panel

\begin{tabular}{|c|c|c|}
\hline Organism & Gene 1 & Gene 2 \\
\hline \multicolumn{3}{|l|}{ Gram Positive } \\
\hline Streptococcus pneumoniae & lyt $A$ & гров \\
\hline Streptococcus agalactiae & sip & \\
\hline Streptococcus pyogenes & speB & \\
\hline Streptococcus spp ${ }^{1}$ & $г р о B$ & \\
\hline Staphylococcus aureus & nuc & гров \\
\hline Coagulase-negative staphylococci $^{2}$ & гроB & \\
\hline Enterococcus spp ${ }^{3}$ & $r p o B$ & \\
\hline Listeria monocytogenes & гров & \\
\hline \multicolumn{3}{|l|}{ Gram-Negative } \\
\hline Escherichia coli & gyr $B$ & ompA \\
\hline Klebsiella oxytoca & gyr $B$ & ompA \\
\hline Klebsiella pneumoniae & gyr $B$ & ompA \\
\hline Enterobacter cloacae & gyr $B$ & ompA \\
\hline Serratia marcescens & gyr $B$ & ompA \\
\hline Enterobacteriaceae $^{4}$ & ompA & \\
\hline Neisseria meningitidis & $\operatorname{ctr} A$ & \\
\hline Haemophilus influenzae & гров & bex $A$ \\
\hline Pseudomonas aeruginosa & gyr $B$ & \\
\hline Acinetobacter baumanii & tus $A$ & $г р о B$ \\
\hline \multicolumn{3}{|l|}{ Yeast } \\
\hline Candida albicans & $\mathrm{ACT}$ & \\
\hline Candida non-albicans 5 & RPB-1 & \\
\hline \multicolumn{3}{|l|}{ Antibiotic Resistance } \\
\hline Methicillin & $\operatorname{mec} A$ & \\
\hline Vancomycin * & $\operatorname{van} A$ & $\operatorname{van} B$ \\
\hline Carbapenem & blaKPC & \\
\hline
\end{tabular}

*anC gene included in panel used for the validation study, but subsequently removed.

${ }^{I}$ Streptococcal species included for primer design were: $S$. pneumoniae, S. parasanguinis, $S$. anginosus, S. salivarius, S. pyogenes, $S$. uberis, $S$. mitis, $S$. sanguis, $S$. mutans, $S$. oralis, $S$. equinis, $S$. gordonii, $S$. gallolyticus

${ }^{2}$ Coagulase-negative staphylococci included for primer design were: S. epidermidis, S. hominis, S. haemolyticus, S. capitis, S. saprophyticus, $S$. warneri, S. xylosus, S. caprae, S. cohnii, S. koosii, S. saccrolyticus, S. nepalensis, S. microti, S. simiae, S. lugdunensis

${ }^{3}$ Enterococcal species included for primer design were: E. facaelis, E. faecium, E. casseliflavus, E. durans, E. gallinarum

${ }^{4}$ Enterobacteriaceae included for primer design were: E.coli, E. hermannii, E. fergusonii, Shigella sonnei, S. boydii, S. dysenteriae, S.flexneri, C. freundii, Salmonella enterica, S. typhimurium, Enterobacter. aerogenes, E. cloacae, E. sakazaki, Klebsiella. oxytoca, K.pneumoniae, Serratia. marcescens 
${ }^{5}$ Non-albicans candida included are: C. parapsilosis, C. glabrata, C. tropicalis, C. krusei 


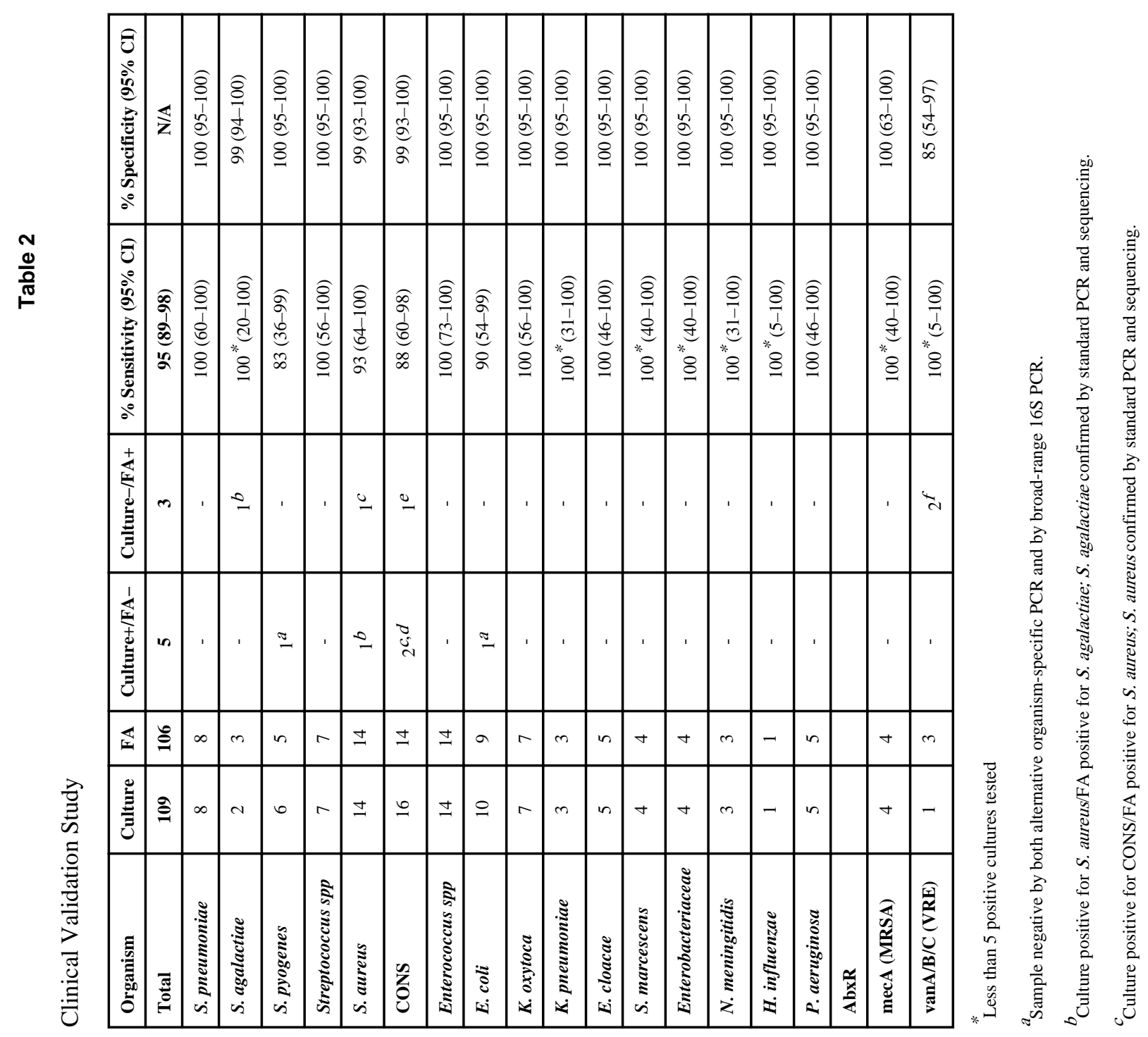




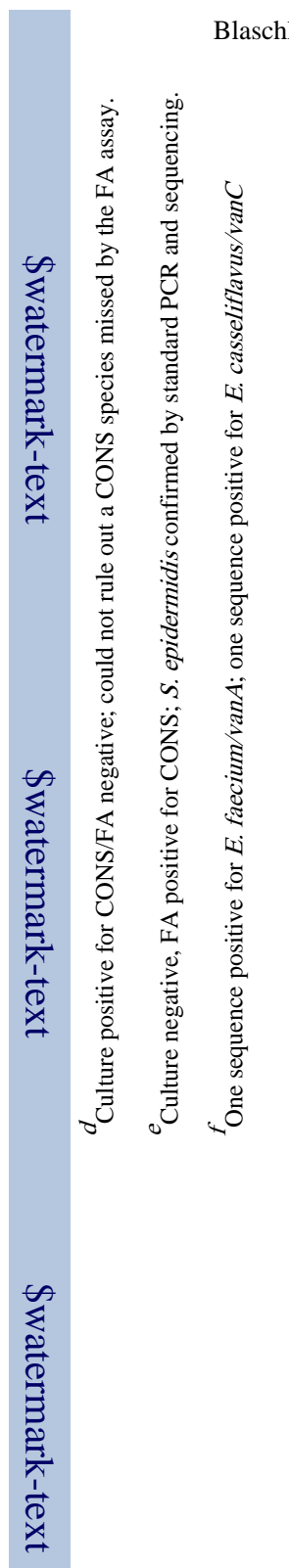

Diagn Microbiol Infect Dis. Author manuscript; available in PMC 2013 December 01. 


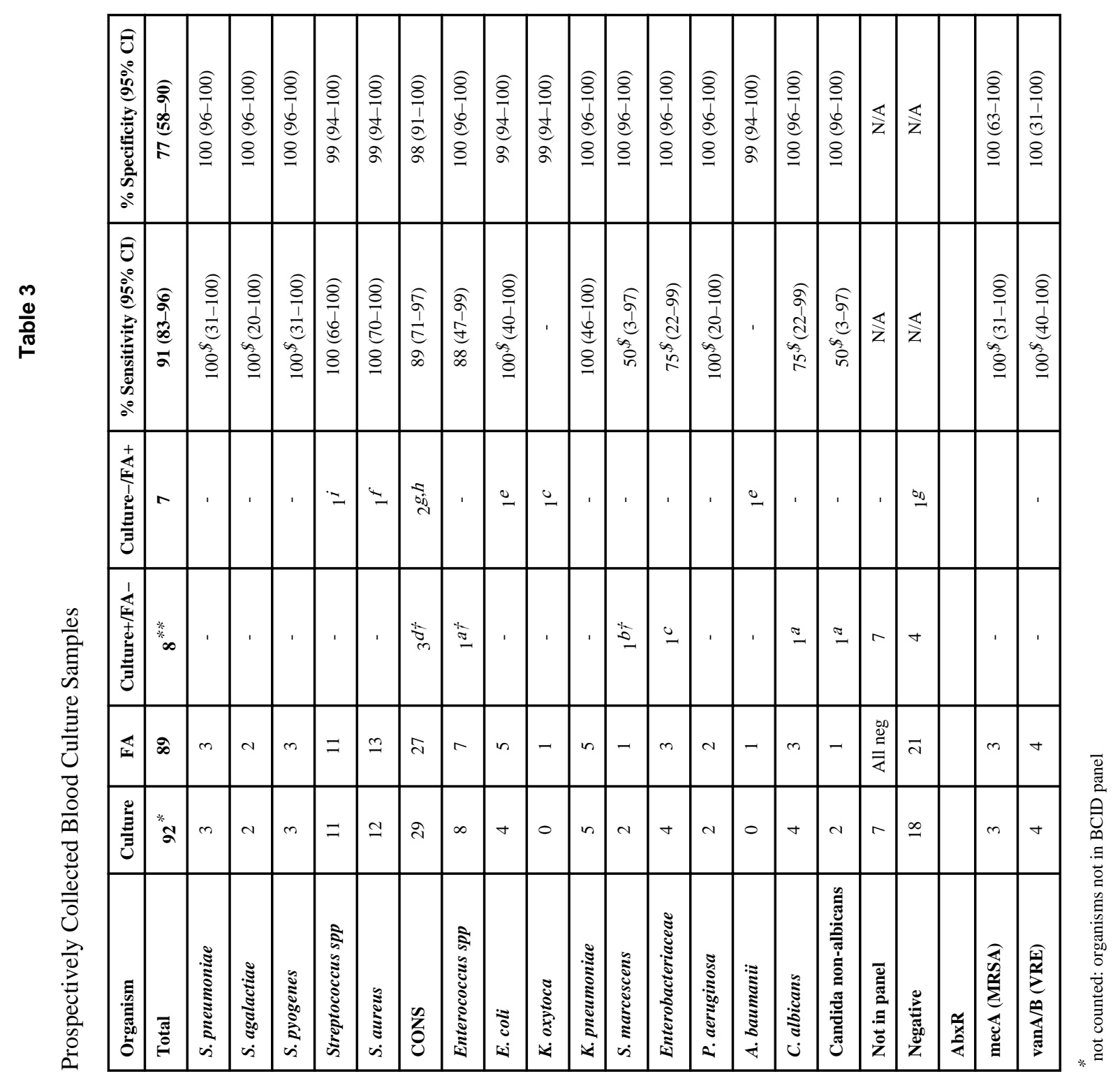




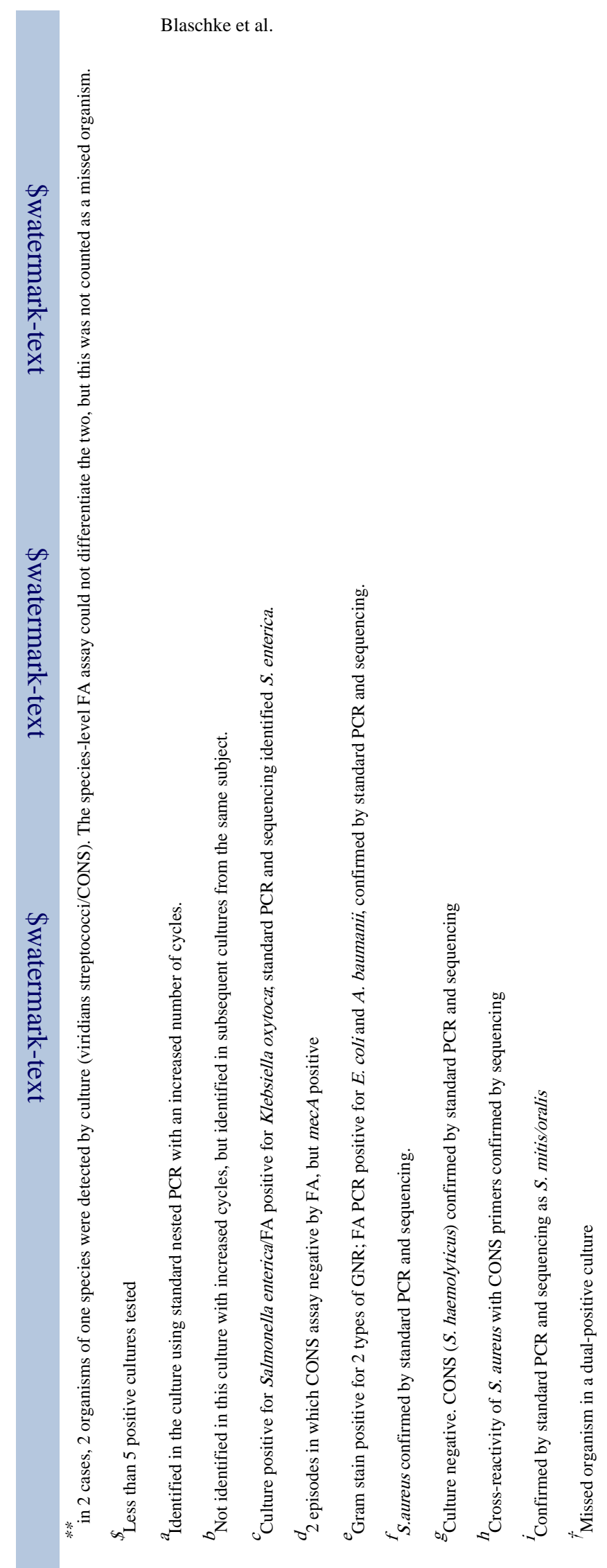

Diagn Microbiol Infect Dis. Author manuscript; available in PMC 2013 December 01. 